Revue internationale P.M.E.

Économie et gestion de la petite et moyenne entreprise

\title{
Financement de la petite et moyenne entreprise à Toumodi (Côte-d'lvoire) : l'illusion informelle
}

\section{Yves-A. Fauré}

Volume 5, numéro 3-4, 1992

URI : https://id.erudit.org/iderudit/1008155ar

DOI : https://doi.org/10.7202/1008155ar

Aller au sommaire du numéro

Éditeur(s)

Presses de l’Université du Québec

ISSN

0776-5436 (imprimé)

1918-9699 (numérique)

Découvrir la revue

Citer cet article

Fauré, Y.-A. (1992). Financement de la petite et moyenne entreprise à Toumodi (Côte-d'lvoire) : l'illusion informelle. Revue internationale P.M.E., 5(3-4), 61-88. https://doi.org/10.7202/1008155ar

\section{Résumé de l'article}

$\mathrm{Au}$ premier rang des préoccupations actuelles de la recherche dans le domaine du financement de la PME africaine, figure, notamment, révaluation des parts occupées par les circuits institutionnels (officiels) et par les réseaux informels, ainsi que l'appréciation du potentiel de soutien dynamique aux activités

d'entreprise représenté par les concours non bancaires. Une enquête menée en Côte-d'Ivoire, auprès de chefs de petites et moyennes entreprises, met en évidence le rôle essentiel joué par l'autofinancement, la rareté des crédits officiels, mais aussi la place très secondaire des concours informels. La nature réelle du processus d'accumulation en vigueur au sein des PME africaines doit conduire en fait à ne pas exagérer l'importance de l'obstacle financier au développement de ces entreprises et, parallèlement, à ne pas fonder de trop grands espoirs sur l'explosion, souhaitée par certains, de l'offre informelle de services financiers. 


\title{
Financement de la petite et moyenne entreprise à Toumodi (Côte-d'Ivoire) : l'illusion informelle
}

\author{
Yves-A. FAURÉ* \\ Centre ORSTOM \\ Ouagadougou
}

\begin{abstract}
RÉSUMÉ
Au premier rang des préoccupations actuelles de la recherche dans le domaine du financement de la PME africaine, figure, notamment, l'évaluation des parts occupées par les circuits institutionnels (officiels) et par les réseaux informels, ainsi que l'appréciation du potentiel de soutien dynamique aux activités d'entreprise représenté par les concours non bancaires. Une enquête menée en Cóte-d'Ivoire, auprès de chefs de petites et moyennes entreprises, met en évidence le rôle essentiel joué par l'autofinancement, la rareté des crédits officiels, mais aussi la place très secondaire des concours informels. La nature réelle du processus d'accumulation en vigueur au sein des PME africaines doit conduire en fait à ne pas exagérer l'importance de l'obstacle financier au développement de ces entreprises et, parallelement, à ne pas fonder de trop grands espoirs sur l'explosion, souhaitée par certains, de l'offre informelle de services financiers.
\end{abstract}

\begin{abstract}
Assessing the proportion of funds provided by official and informal finance networks respectively to small and medium-sized businesses in Africa constitutes one of the main avenues of interest for researchers in the field. Another avenue is to be found in the rating of the possible contribution of nonback financial services to corporate activity.

A survey conducted in the Ivory Coast with the heads of small and mediumsized businesses evidences the importance of self-financing, the dearth of

* $\quad$ Dr Yves-A. Fauré, maître de conférences des Universités, actuellement chercheur, responsable d'un programme de recherche sur l'évaluation des politiques agricoles et le rôle des ONG à l'Institut français de recherche scientifique pour le développement en coopération. A publié plusieurs travaux (articles et ouvrages) de science politique et d'économie politique, notamment sur la Côte-d'Ivoire, dont le dernier : La bataille des entreprises publiques en Côte-d'Ivoire; l'histoire d'un ajustement interne, Paris, Karthala, 1990. Cofondateur de la revue Politique africaine (Paris). Adresse : Centre ORSTOM, 01 BP 182, Ouagadougou, Burkina Faso.
\end{abstract}


institutional/official funds, as well as the secondary role played by informal financial channels.

The very nature of the process by which capital is currently accumulated in small and medium-sized businesses in Africa leads the author to downplay the importance of financial obstacles to their growth and, concurrently, not to place as much hope in the explosion of informal financial services as some would.

\section{RESUMEN}

En el primer rango de las preocupaciónes actuales de la investigación en el dominio de la financiación de la PME africana, encontramos la evalución de las partes que ocupan los circuitos institucionales oficiales y las redes informales, asi como la valoración del potencial de apoyo dinámico a las actividades de empresa representado por las ayudas no bancarias. Una encuesta llevada a cabo en Costa de Marfil, dirigida a los jefes de pecqueñas y medianas empresas muestra la importancia de la autofinanciación, la escasez de créditos oficiales y el papel secundario de las ayudas informales. La naturaleza real del proceso de acumulación en vigor en el seno de las PME africanas conduce a restar importancia al obstáculo financiero para el desarollo de esas empresas en el incremento muy rápido, que desean algunos, de la oferta informal de los servicios financieros.

\section{Introduction}

Longtemps méconnu par les études économiques et sociales relatives à l'Afrique, le monde des activités informelles a fait l'objet d'innombrables analyses à partir des années 70 . Cependant, en dépit de l'intérêt majeur que représentaient ces investigations pour la compréhension des problèmes de développement, en dépit de la justesse des impératifs scientifiques qu'elles servaient en vue de connaître des secteurs d'activité économique souvent prépondérants en termes de contribution aux PIB, en dépit également de l'extrême variété des études réalisées et de l'accumulation des résultats et informations obtenus, les analyses des activités économiques informelles se sont essentiellement concentrées sur deux ou trois thèmes dominants : le marché de l'emploi, la distribution des revenus, le volume des immobilisations. Quant aux vifs et passionnés débats, nés au fur et à mesure que le paradigme informel s'enrichissait de nouveaux travaux, ils ont jusqu'ici portés principalement sur les deux questions de la définition du secteur (approche notionnelle et critériologique) et du type de reproduction qui y prévaut ${ }^{1}$.

1. Pour deux exemples de ces débats, on renverra sélectivement à Charmes (1990) (sur le problème de la définition) et à de Miras (1987) (sur le problème de la reproduction). On n'oubliera pas, en outre, de citer l'article de Le Pape (1983) qui proposait l'une des premières réflexions critiques sur le sens des études informelles. 
C'est tout le mérite des équipes et des chercheurs mobilisés et fédérés par l'Université des réseaux d'expression française (UREF) au sein de l'AUPELF d'avoir à la fois effectué une relance collective des investigations et élargi les thèmes d'analyse des univers économiques informels à la question générale du financement des activités : identification des diverses sources - institutionnelles et non institutionnelles - de formation des capitaux; évaluation de leur part respective à chaque stade de la vie de l'entreprise; repérage des circuits et évaluation des dynamismes financiers non bancaires, de leur potentiel de soutien au développement entrepreneurial ${ }^{2}$.

La faillite des schémas de développement qui confiaient aux États, notamment en Afrique, la charge exclusive de la modernisation sociale et économique, ainsi que l'idée progressivement admise selon laquelle aucune croissance solide et durable ne sera possible sans l'activité d'un secteur entrepreneurial privé dynamique et prospère figurent sans doute parmi les grands déterminants qui inspirent l'intérêt accordé désormais aux financements informels d'entreprise. De même, les échecs avérés des systèmes bancaires officiels, les nécessités d'une mobilisation efficace de l'épargne intérieure pour soutenir des activités économiques échappant désormais à la sphère publique, soit au plan complet de leur organisation soit même au seul plan de leur financement (crédits, subventions, etc.), des circuits financiers publics exsangues et tous contraints par une dette extérieure fort élevée, ont sûrement contribué eux aussi au développement de cet intérêt.

Dans cette nouvelle conjoncture politique, économique et intellectuelle qui tend, d'une part, à moins méconnaître les réalités entrepreneuriales africaines et d'autre part, à élucider les systèmes financiers informels, des progrès significatifs de connaissance ont été accomplis dans le domaine des tontines, ces associations rotatives d'épargnes et de crédit, aux manifestations multiples

2. Il ne s'agit bien en effet, à strictement parler, que d'une relance massive et donc significative d'orientations de recherche déjà abordées dans des travaux ponctuels, extrêmement rigoureux, mais aux résultats faiblement diffusés. On songe, par exemple ici, à l'excellente étude de Benoît Lootvoet (1988) sur les commerçants et artisans de Côte-d'Ivoire, étude qui présente des résultats très précis sur le financement des petits établissements. Le nouvel élan scientifique engagé sous les auspices du Réseau entrepreneuriat de l'UREF/AUPELF s'est concrétisé, par exemple, dans les publications majeures suivantes : l'ouvrage collectif édité sous la direction de Georges Hénault et Rachid M'Rabet (1990) consacré à un tour d'horizon des problèmes de l'entreprise en Afrique francophone, le livre publié sous la direction de Michel Lelart (1990) relatif aux systèmes tontiniers, la série également Notes de recherche du Réseau entrepreneuriat diffusée directement sous le sceau de l'UREF/ AUPELF et qui, depuis 1989, a recueilli plus d'une trentaine d'études portant sur les financements et circuits financiers informels. 
dans le monde, mais toutes assises sur des pratiques sociales solides et anciennes. Sous l'égide de l'UREF/AUPELF, de nombreuses études ont décrit avec minutie les systèmes tontiniers en vigueur au Cameroun, au Sénégal, au Niger, au Bénin, au Togo, etc., pour ce qui concerne le seul espace francophone africain sub-saharien. Ces travaux, qui ont l'avantage de lever enfin le voile sur des phénomènes largement répandus, mais jusqu'ici peu analysés, permettent d'en expliciter les mécanismes techniques, d'en comprendre les contextes sociaux et de suggérer enfin, l'importance - en toute hypothèse considérable - des pratiques financières informelles.

Cependant, si ces études, la plupart produites récemment, permettent de faire reculer chaque fois davantage les obscurités et les mystères d'un monde resté jusque-là "souterrain » ou "parallèle », elles ne règlent pas pour autant l'ensemble des problèmes que pose la finance informelle sous l'angle du développement en général et de l'épanouissement d'un milieu entrepreneurial solide, en particulier. En premier lieu, si les tontines représentent les manifestations les plus spectaculaires des pratiques financières populaires, celles-ci empruntent s'il est permis d'utiliser un tel terme sur le sujet ! - d'autres formes telles que les activités de gestion des dépôts assurées par les innombrables banquiers de rue des villes africaines et gardes-monnaie villageois ou encore les formules d'avances et de prêts - plus rarement de dons - consentis dans les réseaux lignagers et les configurations communautaires. En s'en tenant à ces seuls exemples parmi d'autres, le tableau des circuits financiers non institutionnels devient sensiblement plus complexe et plus diversifié. Seul un bilan intégrant l'ensemble des pratiques financières informelles en restituera la richesse, car les situations pratiques des ménages et des entrepreneurs se caractérisent couramment par des combinaisons de plusieurs techniques informelles.

En outre, les nombreuses études sur les systèmes financiers informels n'éclairent encore que très modestement la question de leurs fonctions dans la capitalisation des entreprises. D'une manière générale, la part relative occupée par le financement des petits et moyens établissements de production, de services et de commerce dans l'ensemble des flux financiers informels et autres réseaux populaires de mobilisation d'épargne demande encore à être mesurée précisément. Quelle est l'importance des apports non bancaires dans la formation du capital initial et dans les investissements complémentaires éventuellement réalisés au cours du cycle de croissance de l'entreprise ? Peut-on induire de l'étendue et de la multiplicité des pratiques financières informelles l'ampleur des sources non institutionnelles de capitaux des entreprises petites et moyennes en Afrique sub-saharienne? Les systèmes tontiniers si répandus dans le souscontinent remplissent-ils un rôle clair et net dans le montage financier des affaires, les fonds qu'ils servent sont-ils utilisés, et jusqu'à quel point, à des fins d'entreprises, au financement de leurs activités économiques? La rareté, voire 
l'impossibilité d'accès au crédit bancaire constatées dans la plupart des études des PME en Afrique sub-saharienne sont-elles compensées par une mobilisation financière sur des bases informelles? Les « problèmes d'argent », souvent évoqués par les petits entrepreneurs comme étant parmi leurs plus pressantes préoccupations, signifient-ils que des solutions sont recherchées et trouvées par eux dans les circuits et dans des formules non institutionnelles? Telles sont quelques-unes des questions que la littérature, à présent disponible sur le sujet, laisse encore en suspens.

Le présent texte a pour modeste ambition d'apporter de premières réponses précises à ces questions en rendant compte des résultats d'une recherche de terrain réalisée dans une ville moyenne de Côte-d'Ivoire. Les conditions de conduite des investigations, les caractéristiques de la population entrepreneuriale enquêtée donnent à penser que les résultats obtenus, sans être rigoureusement et strictement extrapolables, reflètent cependant assez bien la situation financière des petites et moyennes entreprises pour l'ensemble de la Côte-d'Ivoire. Ces résultats tendent à tempérer les trop fortes attentes qui pèseraient sur les systèmes informels - notamment tontiniers - pour venir au secours des PME africaines en mal de financement. Les réalités économiques et sociales, objectivement restituables par l'analyse, ne doivent pas forcément conduire à "désespérer l'informel financier », mais elles incitent à ne pas sombrer dans un enchantement anthropologique qui verrait hâtivement, dans le génie populaire financier, la clef de la prospérité économique des entreprises et, partant, la solution à certains obstacles au développement.

Les conditions de l'enquête et les caractéristiques principales de la population des petits entrepreneurs observés ne peuvent être que très brièvement évoquées dans le cadre étroit de ce document ${ }^{3}$. On se contentera de signaler que l'enquête a concerné des chefs d'entreprises relevant des activités non agricoles (production artisanale et semi-industrielle, services, commerce) et exerçant dans des locaux professionnels, que les points d'activité informelle ont été exclus, et que les établissements retenus correspondent à la classe des petites entreprises et à la frange inférieure des moyennes entreprises. Constitué sur la technique des quotas (sexe, nationalité, branches et sous-branches, taille, etc.) et stratifié

3. Le concours du Réseau entrepreneuriat de l'UREF/AUPELF, coordonné par Bruno Ponson, a contribué au financement du traitement informatique des données d'enquête de Toumodi. Les résultats complets de l'enquête de Toumodi sont disponibles dans l'étude, publiée sous le même titre, dans la série Notes de recherche $n^{\circ} 92.25$ du Réseau entrepreneuriat de l'UREF/AUPELF. Quant aux analyses plus globales entrant dans le cadre du programme de recherche conduit sur l'ensemble de la Côted'Ivoire au titre de l'ORSTOM (Toumodi, Daoukro et Abidjan), elles font l'objet d'un rapport général, diffusé sous le sceau de cet institut à la fin de l'année 1992. 
par zones géographiques urbaines, l'échantillon enquêté a porté sur 106 entrepreneurs.

Les 106 entrepreneurs enquêtés à Toumodi exercent 35 types d'activités différentes correspondant à 11 branches de la nomenclature de la comptabilité nationale et se répandent ainsi dans les trois grands secteurs de l'économie : 24 $(23 \%)$ dans la production (artisanale ou semi-industrielle), 33 (31\%) dans les services et $49(46 \%)$ dans le commerce. Cette distribution est à peu près conforme aux recensements disponibles par ailleurs et aux études faites par d'autres économistes. Ces 106 entreprises fournissent chacune en moyenne 3 emplois, puisqu'on y dénombre 112 patrons, 36 salariés permanents, 97 apprentis et 59 aides familiales. Les entrepreneurs, en totalité, sont assujettis aux principaux impôts assis sur l'activité économique : 100 relèvent de la petite patente municipale acquittée mensuellement auprès des services financiers communaux et 6 émargent à la grande patente due à la Direction générale des impôts. Sur ces 106 entrepreneurs, $78 \%$ sont des hommes et $22 \%$ des femmes ; ce qui correspond là encore, compte tenu du type d'entreprises observées, à une ventilation vérifiée à l'échelle du pays. Illustration des mouvements de forte immigration qu'a connus la Côte-d'Ivoire, du fait de taux élevés de croissance économique jusqu'au début des années 80 , la moitié de l'échantillon est constituée d'Africains non Ivoiriens. Au point de vue de l'âge, la classe modale est de 30-39 ans ( $45 \%$ de l'effectif total), celle des $40-49$ ans comptant $24 \%$ des enquêtés et celle des 20-29 ans, $21 \%$. Ce sont $39 \%$ des entrepreneurs qui sont propriétaires de leur local tandis que $55 \%$ d'entre eux le louent et que $6 \%$ l'utilisent gracieusement. Enfin, $88 \%$ des entrepreneurs enquêtés tirent un revenu net moyen mensuel oscillant entre 25000 et 99000 FCFA qui doit être prudemment comparé au niveau de salaire minimum interprofessionnel garanti en vigueur au moment des investigations (35000 FCFA/mois).

Ces très rapides précisions étant apportées, il convient de s'arrêter désormais sur les résultats des investigations financières obtenus à l'issue de cette enquête et de proposer une interprétation qui rende intelligible leurs orientations majeures.

\section{La diversité diachronique des modes de financement}

La dimension financière des entreprises, dont on vient de présenter quelques caractéristiques générales, a été attentivement examinée lors des longues enquêtes de terrain à travers plusieurs moments décisifs dans la vie des affaires et sous les diverses formes et composantes qu'elle peut successivement ou simultanément revêtir. La formation du capital de départ, le financement des 
investissements ultérieurs et complémentaires, ainsi que les solutions apportées aux problèmes de trésorerie, ont notamment fait l'objet de patientes explorations et mesures.

\subsection{La formation du capital initial}

Premier acte essentiel de la vie de l'entreprise, la constitution du capital initial mérite la plus grande attention. Les différentes sources ayant permis la mobilisation des fonds de départ apparaissent clairement dans le tableau 1. L'épargne personnelle accumulée antérieurement à la présente activité entrepreneuriale enquêtée est vérifiée auprès de 62 exploitants sur 106, cependant que les prêts financiers en ont concerné 28 sur 106 et les dons, 21 sur 106. En première analyse, les efforts monétaires consentis directement par l'entrepreneur constituent, et de loin, la première source de formation du capital initial.

TABLEAU 1

Sources du capital initial

\begin{tabular}{llrrlcc}
\hline Sources individualisées & Effectifs & $\%$ & Sources combinées & Effectifs & $\%$ \\
\hline Épargne & Oui & 62 & 58 & Épargne & 53 & 50 \\
personnelle & Non & 44 & 42 & Prêt & 21 & 20 \\
& Total & 106 & 100 & Don & 20 & 18,5 \\
\hline Don & Oui & 21 & 20 & Prêt + épargne & 7 & 6,5 \\
& Non & 85 & 80 & Autre source & 3 & 3 \\
& Total & 106 & 100 & 1 & 1 & \\
\hline Prêt & Oui & 28 & 26 & Autre source + épargne & 1 & 1 \\
& Non & 78 & 74 & Prêt + don & 0 & 0 \\
& Total & 106 & 100 & & & \\
\hline \multirow{2}{*}{ Autre source } & Oui & 4 & 4 & Épargne + prêt + don & & \\
& Non & 102 & 96 & & 106 & 100 \\
\hline
\end{tabular}

Mais l'analyse demande à être poussée plus avant pour mieux détecter et pondérer, au sein des différentes sources de constitution du capital de départ, les pratiques formelles et informelles. On relèvera tout d'abord que les montants mobilisés dans les trois sources principales connaissent de grandes amplitudes : les 62 cas sur 106 concernés par l'épargne personnelle se répartissent en 8 classes allant de plus de 5000 FCFA à plus d'un million, et 40 cas sur les 62 
(soit $65 \%$ ) émargent aux classes intermédiaires (de 25000 FCFA à 499000 FCFA) ; les cas de prêts (28/106) dont les montants ont pu être précisés s'échelonnent sur 5 classes (de 25000 FCFA à plus d'un million); les situations de dons (21/106) se ventilent sur 7 classes, mais correspondent, en majorité, à de faibles montants. D'autre part, les trois grandes sources de financement relevées ici sont inégalement réparties selon les diverses caractéristiques des entreprises et entrepreneurs concernés : alors qu'elles constituent $22 \%$ de l'échantillon enquêté, les femmes sont sous-représentées dans la formule « épargne » $(15 \%)$ et dans la formule "prêt» $(11 \%)$, alors qu'elles sont nettement surreprésentées dans la formule «don » $(48 \%)$. D'autre part, aucune des trois sources particulières de formation du capital initial n'induit de résultats financiers particuliers : le croisement du type de source et de la variable "classes de revenus nets obtenus par l'entrepreneur » montre une grande dispersion des effectifs concernés par l'épargne, le prêt et le don sur l'ensemble des valeurs de l'échelle (tableau 2).

\section{TABLEAU 2}

\section{Structure des caractéristiques d'entreprise selon différentes sources de constitution du capital initial}

\begin{tabular}{|c|c|c|c|c|c|c|c|c|c|c|}
\hline Sexe & Hommes & \multicolumn{2}{|c|}{ Femmes } & Total & Secteur & Production & Services & \multicolumn{2}{|c|}{ Commerce } & Total \\
\hline Épargne & $85 \%$ & & $\%$ & $100 \%$ & Épargne & $24 \%$ & $23 \%$ & & $\%$ & $100 \%$ \\
\hline Prêt & $89 \%$ & & $\%$ & $100 \%$ & Prêt & $18 \%$ & $29 \%$ & & $\%$ & $100 \%$ \\
\hline Don & $52 \%$ & & $\%$ & $100 \%$ & Don & $29 \%$ & $52 \%$ & & $\%$ & $100 \%$ \\
\hline Résultat & \multicolumn{10}{|c|}{ Classe de revenus nets en milliers de FCFA/mois } \\
\hline financier & $\begin{array}{l}\text { moins } \\
\text { de } 10\end{array}$ & 25 à 49 & 50 à 74 & 75 à 99 & $\begin{array}{c}100 a ̀ \\
149\end{array}$ & $\begin{array}{l}150 \text { aे } \\
249\end{array}$ & $\begin{array}{c}250 \text { à } \\
500\end{array}$ & $\begin{array}{l}\text { plus de } \\
500\end{array}$ & $\begin{array}{c}\text { non } \\
\text { précisé }\end{array}$ & Total \\
\hline Épargne & $1,60 \%$ & $27,4 \%$ & $29,0 \%$ & $33,9 \%$ & & $3,2 \%$ & & $1,6 \%$ & $3,2 \%$ & $100 \%$ \\
\hline Prêt & & $32,1 \%$ & $35,7 \%$ & $14,3 \%$ & & & $7,1 \%$ & & $10,7 \%$ & $100 \%$ \\
\hline Don & & $38,1 \%$ & $23,8 \%$ & $23,8 \%$ & $4,8 \%$ & $9,5 \%$ & & & & $100 \%$ \\
\hline
\end{tabular}

Note : Ce tableau porte sur un effectif total de 103 entrepreneurs se décomposant en 94 entrepreneurs ayant mobilisé une source unique d'investissement initial (53, «épargne », 21, « prêt »; 20 , « don ») auxquels ont été ajoutés 9 entrepreneurs ayant eu recours à des sources combinées (ex., épargne + prêt) et pour lesquels une source dominante est clairement apparue dans le financement de départ. Ces 9 entrepreneurs ont été classés dans les catégories concernées (« épargne », « prêt » ou « don »).

S'agissant plus particulièrement des caractéristiques économiques de l'épargne personnelle à l'origine de la création de l'entreprise alors enquêtée à Toumodi, on notera que les 62 cas enregistrés ( $58 \%$ du total) se distribuent en 
20 cas d'accumulation de revenus salariés et en 42 cas de revenus d'une précédente activité - souvent de moindre importance ou localisée ailleurs ou encore, exercée dans un autre secteur - sur ces 42 derniers cas, 9 concernent une épargne constituée précédemment dans l'activité artisanale ; 6, dans les services ; 27, enfin, dans le commerce. L'épargne constituée préalablement provient donc à hauteur de $68 \%$ d'une activité antérieure d'entreprise, ce qui suggère bien, relativement au type d'entrepreneurs enquêtés (PME assez structurées), un processus d'autofinancement des activités économiques privées relativement important et, en tout état de cause, dominant. Ce fait dénombre même les possibilités d'accumulation qu'offrent, à un certain stade, les activités commerciales et artisanales urbaines trop souvent vues comme assurant à peine la survie des exploitants. Des tendances semblables étaient déjà notées dans les résultats des travaux de Lootvoet (Lootvoet, 1988 : 198-205).

Aux 28 entrepreneurs ( $26 \%$ ) bénéficiaires d'un prêt financier lors du démarrage de leur activité présentement enquêtée et signalée dans le tableau 1 , ont été ajoutés, pour les besoins de l'analyse économique et non pas strictement financière, 23 autres qui ont reçu un prêt sous forme physique (équipement : machine, outils ; fourniture de stock : la majorité). Ce sont donc en réalité 51 entrepreneurs sur 106 (48\%) qui ont profité de cette source, monétaire ou non monétaire, pour créer leur activité actuelle, un seul cas de prêt combiné (financier et physique) ayant été relevé. L'analyse des conditions de ces différents prêts permet de conclure que dans 29 cas sur 51 , le fournisseur intervient, la famille dans 16 cas, le réseau d'amis dans 3 cas, la banque dans 2 cas. On notera que pas une seule fois l'ancien employeur ou un quelconque banquier non institutionnel et autre prêteur sur gage ne sont évoqués ; l'association tontinière apparaît une fois.

Une étude fine des prêts obtenus à Toumodi indique que ceux-ci, appréciés à l'aune de plusieurs indicateurs (durée, garanties, régularité et rythme de remboursement, etc.) peuvent être considérés comme ayant été contraignants dans 44 cas sur 51, ce qui explique que tous les entrepreneurs soient devenus, depuis la création de leur établissement, propriétaires de leurs moyens de production. En outre, ces résultats s'inscrivent contre une tendance consistant à présenter les prêts, dans les sociétés africaines, comme des instruments financiers souples, voire «mous ", générant des obligations de remboursement flexibles, négociables, ceci conformément à une vision impliquant un fonctionnement en permanence transactionnel des relations sociales au sein d'ensembles « communautaires » fondés sur des solidarités agissantes, etc.

Quant aux dons obtenus par $20 \%$ des entrepreneurs (21 cas), ils se présentent en grande majorité sous forme monétaire (19 cas). Ils proviennent exclusivement du cercle familial $(88 \%)$ ou d'un ancien employeur. De patientes 
investigations sur cette question permettent d'avancer que, dans un cas sur deux, le don, formellement présenté et ressenti ainsi, s'accompagne en réalité de contreparties contraignantes de la part du récipiendaire (obligation d'embauche, d'aide et assistance financière, etc.). En conséquence, on dira que les dons ne sont pas aussi importants que le laisserait croire une vue idéalisée des sociétés africaines et, en second lieu, que des dons peuvent en fait cacher d'autres formes de relations d'affaires (prêts déguisés, avances sournoises, etc.). C'est sans doute ce qui explique des différences de résultats assez sensibles entre la présente recherche - où un contrôle constant et vigilant a été opéré sur les différentes sources de capital et, à l'intérieur de celles-ci, sur les conditions réellement consenties aux entrepreneurs - et d'autres enquêtes qui signalent une importance plus grande aux dons dans les origines du capital initial des entreprises, (par exemple, Lootvoet $(1988: 198)$ qui a enregistré l'existence de dons dans presque un cas sur deux de création des petites activités ; il est vrai, en outre, que son échantillon comprend une proportion importante de femmes et que, on l'a vu plus haut, celles-ci, plus que les hommes, bénéficient de ce type d'apport initial.

\subsection{Le financement des investissements ultérieurs}

L'effort de capitalisation ne s'arrête pas avec le montage financier initial de l'entreprise. En cours d'activité, dans le cycle de croissance de l'entreprise, des investissements sont réalisés. Nos observations toumodiennes renforcent des tendances déjà signalées par maintes études : peu d'entrepreneurs agrandissent et modernisent le local professionnel - dont ils ne sont pas, on l'a vu, majoritairement propriétaires, ceci expliquant sans doute cela ; en revanche, en dehors des utilisations «sociales » des surplus financiers dégagés de l'activité (nourrir, héberger, éduquer, construire une habitation, etc.) des investissements sont pratiqués sous forme de renouvellement/amélioration de l'équipement en machines et en outils ou d'augmentation des stocks de marchandises ou de matières premières. Si de tels investissements complémentaires ne sont pas forcément très importants ou systématiquement réalisés, ils sont assez courants pour être signalés ici et analysés dans leur procédure.

Ce sont les revenus tirés de l'activité entrepreneuriale elle-même qui financent en priorité ces investissements ultérieurs pour 94 des 106 exploitants interrogés. Toutefois, 34 entrepreneurs ont complété leur autofinancement par des prêts (dont 3 cas de tontine) et 2 autres par des dons, un seul ayant par ailleurs déclaré avoir utilisé les revenus d'une autre activité simultanément exercée. On voit que la hiérarchie des sources de constitution du capital initial est respectée. Le réinvestissement, tout en restant, insistons sur ce point, de faible volume, représente cependant la procédure essentielle d'accroissement de la 
capacité de production. Dans les cas de financement par des prêts - qui ne font au reste que s'ajouter à l'autofinancement - il s'agit de manière prédominante de prêts consentis par les fournisseurs (comme dans la période initiale). Les prêts bancaires sont absents, où ils prennent la forme de prêts à la consommation. Il n'est pas fait appel à d'autres formules (revenus locatifs, fonds réunis dans des tontines, etc.).

\subsection{Les problèmes de trésorerie}

Seuls $8 \%$ des 106 entrepreneurs de Toumodi constituant l'échantillon d'enquête ne semblent jamais avoir connu de difficultés financières après le lancement de l'activité. Des problèmes de gestion à court terme ont surgi dans le cycle de vie de la plupart des entreprises observées. La très grande majorité des exploitants n'a pas fait, même occasionnellement, appel à des fonds extérieurs, se contentant alors de réduire leurs ponctions et enregistrant conjoncturellement de fortes tensions dans leur trésorerie. Dans $6 \%$ des cas, les exploitants n'ont pas hésité à recourir à une avance de la clientèle pour faire face à une situation financière délicate. Aucun cas de recours à des usuriers et autres bailleurs informels ou à une tontine n'a été observé.

Les résultats obtenus à Toumodi sur ce chapitre sont conformes à ceux des autres enquêtes conduites en d'autres endroits de Côte-d'Ivoire dans le cadre de la même grande opération de recherche. Ainsi, pour ne retenir que cet exemple, à Daoukro, aucun des 280 petits et moyens entrepreneurs interrogés ne semble avoir eu recours, pour régler des problèmes financiers à court terme, à d'autres formules que des avances-clients et des avances-fournisseurs, la plupart gérant ces difficultés conjoncturelles en tendant davantage la trésorerie de l'entreprise et en modulant les prélèvements opérés quotidiennement sur les recettes pour couvrir les dépenses courantes d'entretien de l'unité de résidence.

Il ne doit cependant pas exclure que des régulations financières soient établies au sein de l'ensemble des sources de revenus et des activités exercées par les exploitants, mouvements accentués par une situation de faible - mais non nulle comme on le croit trop souvent - séparation des patrimoines (entre le domestique et le professionnel ou encore entre ceux relatifs à des activités différentes, mais simultanées). Aucune mention n'a été faite par les entrepreneurs, pourtant interrogés longuement, de l'existence de tels transferts propres à soulager la trésorerie de l'entreprise dans des périodes difficiles. Cependant, 21 entrepreneurs sur 106 font état d'autres sources de revenus. Mais ces transferts ne peuvent être très importants. En effet, seuls 4 entrepreneurs sur 106 perçoivent des revenus locatifs, 11 disposent de très modestes revenus agricoles (commercialisation de vivriers essentiellement), les 6 autres pouvant bénéficier des quelques gains tirés par le conjoint dans une micro-activité informelle. C'est 
l'exercice d'une multi-activité $(23 \%$ des entrepreneurs de Toumodi sont propriétaires d'au moins une deuxième entreprise) qui peut surtout donner à penser que des transferts de trésorerie sont éventuellement opérés, puisque les 24 exploitants concernés ont déclaré tirer des revenus de cette autre ou de ces autres activités patronales. Ces circulations financières sont largement plausibles et peuvent correspondre, au plan de la trésorerie, au processus endogène de financement de nouvelles entreprises déjà noté à propos des sources du capital initial. Une réserve doit cependant être apportée : la moitié des entrepreneurs ayant une autre entreprise l'ont ailleurs qu'à Toumodi, rendant plus aléatoires les mouvements de fonds entre les divers établissements d'un même propriétaire.

\section{La place relative des financements informels}

Avant d'aller plus loin dans l'analyse, il est bon de rassembler, sur une base cette fois typologique et non plus évolutive, les principaux résultats détaillés dans les développements précédents. Il s'agit en effet de mieux identifier et d'évaluer la part des pratiques informelles dans l'ensemble des modalités financières auxquelles ont recours les petits et moyens entrepreneurs. Sans être négligeable, on verra que cette part est assez marginale.

\subsection{La hiérarchie des sources de financement à Toumodi}

On aura noté en premier lieu l'importance majeure de l'autofinancement. Celuici se présente sous la forme d'une épargne personnelle, vérifiée chez $58 \%$ des promoteurs lors de la création de l'entreprise, et elle-même provenant pour l'essentiel $(68 \%)$ d'une activité entrepreneuriale préalable. L'importance des fonds propres apparaît également dans le financement des investissements additionnels alimentés chez $89 \%$ des entrepreneurs par les bénéfices qu'ils parviennent à dégager de la présente activité enquêtée.

Le recours à des prêts strictement financiers lors de la création de l'entreprise concerne $26 \%$ des promoteurs. L'essentiel de ceux-ci viennent des fournisseurs (pour $57 \%$ des cas étudiés, et prennent alors l'aspect d'une avance de marchandises ou de matières premières) ou du cercle familial (pour $31 \%$ des situations concernées par un prêt). Au total, deux cas de prêts consentis par les banques ont été signalés par les 106 entrepreneurs interrogés. Le concours financier d'une tontine apparaît également dans le cas d'une création dans l'ensemble de l'échantillon d'enquête. En matière d'investissements complémentaires, la formule du prêt est venue compléter, dans $32 \%$ des situations, la 
réinjection des bénéfices de l'entreprise. Sur les 34 cas considérés, on relève 31 prêts de fournisseurs et les tontines ont été mises à contribution dans trois cas.

Les dons, enfin, sont présents dans $20 \%$ des situations de création d'entreprise et proviennent du cercle familial dans $88 \%$ des cas et, pour le reste, d'anciens employeurs. Dans le domaine des investissements ultérieurement réalisés par les entrepreneurs, les dons ne sont repérés que dans $2 \%$ des cas.

Sous le rapport de plus ou moins grande institutionnalité, de plus ou moins grande formalité des modes de financement auxquels ont recours les entrepreneurs de Toumodi, il est possible de tirer rapidement les quelques enseignements suivants.

Le système bancaire manifeste une très grande discrétion. Ses concours financiers sont rares : deux seuls cas ont été relevés dans la diversité des 106 créations d'entreprise et aucune intervention du circuit financier institutionnel n'a été notée lors des investissements complémentaires. Encore convient-il de préciser que dans les deux situations évoquées ici, les promoteurs ont obtenu un crédit à la consommation sous forme de «prêt personnel » complétant leur propre mise de fonds au lieu d'un crédit commercial (les prêts personnels sont remboursables sur dix mois); $90 \%$ des crédits consentis dans ce cadre par les trois agences bancaires de la ville se situent entre 500000 et 1000000 FCFA.

Ce résultat est d'autant plus remarquable qu'environ $30 \%$ des entrepreneurs enquêtés disposent pourtant d'un compte dans une des agences bancaires de Toumodi. Les établissements financiers sont en effet bien présents dans la ville à travers la SGBCI, la $\mathrm{BNDA}$ et la $\mathrm{BICICI}$ et gèrent, au moment de l'enquête, respectivement 1500,650 et 1700 comptes, soit près de 3900 comptes au total. Mais, alors que les "produits» proposés par les banques sont diversifiés (compte-courant, compte-livret, plan d'épargne-logement et plan d'épargne-crédit, dépôt à terme, etc.), celles-ci ne gèrent que très peu de « comptes commerciaux ». La plupart des entrepreneurs disposent simplement de " comptes de particuliers » et les entretiens réalisés auprès des trois directeurs d'agences bancaires confirment pleinement la rareté des prêts consentis aux PME de Toumodi apparue dans les enquêtes d'entreprises. Il est, à cet égard, significatif que la plus grande agence n'ait financé que les deux pharmacies de la place et que la troisième en importance n'ait accordé au total qu'un seul crédit commercial dont a bénéficié un artisan-sculpteur (ces trois établissements ne sont pas inclus dans notre échantillon d'enquête). Seuls quelques solides exploitants sollicitent et obtiennent des «facilités de caisse», d'ailleurs chèrement payées par d'importants agios trimestriels. Les banques ne participent donc pas au financement des entreprises à Toumodi. 
Le chapitre des financements informels paraît davantage nourri par les résultats de l'enquête, mais la prudence analytique s'impose tout autant. Le principal pourvoyeur semble être constitué par la communauté lignagère qui manifeste sa participation, on l'a vu, soit sous forme de prêts, soit sous forme de dons à l'occasion du lancement de l'activité beaucoup plus que lors des investissements ultérieurs dont elle est quasiment absente. Faut-il encore préciser que, sur le plan des seuls apports financiers, les prêts familiaux ne concernent finalement que $31 \%$ des $26 \%$ des promoteurs ayant bénéficié de prêts financiers de toutes origines au moment de la création de l'entreprise, soit environ $8 \%$ du total des entrepreneurs enquêtés, et que les fonds fournis par le cercle familial à ce même stade de création de l'entreprise ne concernent qu'environ $15 \%$ de l'ensemble des promoteurs. À ces chiffres qui viennent relativiser la contribution lignagère dans le lancement des activités, il faut ajouter deux précisions qui nuancent encore davantage l'ampleur des concours familiaux. En premier lieu, on l'a vu, les prêts consentis dans ce cadre peuvent être considérés comme contraignants pour $86 \%$ d'entre eux ; d'autre part, les dons sont souvent assortis de contreparties qui leur ôtent les caractéristiques d'apports gracieux, indolores, urilatéraux dont on les affuble trop rapidement sous prétexte que ces aides proviennent de foyers de solidarité primaire.

On aura pu noter également l'absence de bailleurs informels auxquels on songe habituellement : banquier de rue, garde-monnaie, prêteur sur gage, etc. Il faut relever ici la grande sagesse gestionnaire des petits et moyens entrepreneurs de Toumodi méfiants à l'égard des financements de proximité, certes facilement débloqués, mais dont les taux d'intérêt sont loin d'être mesurés. Ceci est d'autant plus notable que les prêteurs sur gage, dans une ville comme Toumodi (où ils sont appelés "maraka » et qui sont d'origine malienne), sont nombreux et prospères, qu'ils offrent "généreusement » leurs services dans les arrières boutiques des quartiers commerciaux et qu'ils sont, au total, autrement disponibles et "accueillants » que les agences des trois établissements bancai-. res officiels présents à Toumodi. Ils pratiquent certes des taux usuriers (de l'ordre de $100 \%$ par mois selon nos observations de terrain), mais leurs services sont immédiats. Ils interviennent financièrement selon deux types de modalités : soit le prêt d'argent direct pratiqué à des taux d'intérêt, on l'a vu, considérables, soit le rachat de dette contractée lors d'un achat d'un bien d'équipement, souvent le seul ouvert aux particuliers. Est-il nécessaire d'insister sur le caractère asphyxiant d'un tel mécanisme financier pour ceux qui y ont recours ? Les exploitants des PME de Toumodi ne règlent cependant pas de cette façon leurs problèmes financiers immédiats - $\mathbf{a}$ fortiori la question du capital permanent de l'entreprise - et, de fait, l'enquête montre que ce sont les salariés régulièrement rémunérés du secteur privé moderne, ainsi que les agents des secteurs étatique et parapublic, qui sont les catégories socioprofessionnelles les plus 
engagées auprès des circuits financiers parallèles, n'hésitant pas à gager plusieurs mois de revenus à venir.

Quant au système tontinier, sa contribution se résume à un seul apport sur les 106 créations d'entreprises et à 3 concours dans des opérations d'investissements ultérieurs. Il est vrai que les participations à des associations rotatives d'épargne et de crédit n'ont été déclarées que par $4 \%$ des effectifs enquêtés. Cette faiblesse s'explique sans doute par la composition de l'échantillon : $22 \%$ de femmes entrepreneurs y figurent alors que les tontines, en Côte-d'Ivoire, sont nettement féminisées $(22 \%$ des 60 femmes chefs d'entreprises enquêtées à Abidjan y adhèrent régulièrement). De fait, lorsqu'on analyse les participations à des tontines faites par les entrepreneurs enquêtés, on enregistre que les 4 personnes concernées sont toutes des femmes, que 3 travaillent dans le commerce et 1 dans les activités de service, que 2 appartiennent à la classe d'âge 40-49 ans, 1 à la classe 30-39 ans, la dernière à la classe 50-59 ans. En termes de revenus nets tirés de l'activité, ces 4 femmes participant à des tontines émargent aux 4 classes les plus faibles sur les 9 statistiquement construites.

On peut encore préciser le profil de ces 4 tontinières et des conditions d'emploi des fonds collectés. Madame A., 52 ans, dyula, tient un commerce de pagnes et la tontine lui a permis un apport de 120000 FCFA en début d'activité. Madame B., 31 ans, yoruba originaire du Nigéria, exploite un magasin de récipients divers (terre, plastique, etc.). En cours d'activité, les 200000 FCFA mobilisés dans le cadre d'une tontine ont contribué au financement de l'agrandissement du local. Madame C., 44 ans, baoulé, a utilisé 40000 FCFA en provenance d'une tontine pour développer ses activités de maquis-restaurant. Enfin, Madame D., 41 ans, baoulé, a orienté les 200000 FCFA provenant d'une tontine dans l'achat de machines supplémentaires pour son entreprise de confection et de vente de vêtements d'enfants.

\subsection{De la situation locale à la situation générale}

Les résultats ainsi obtenus sur Toumodi en matière de mode de financement des entreprises posent la légitime question de leur particularité locale ou, au contraire, de leur conformité à des situations prévalant ailleurs, en Côte-d'Ivoire ou en Afrique sub-saharienne, et décrites par maintes études. Mais de telles comparaisons, sans doute indispensables pour apprécier l'aire de validité des données issues de l'enquête de terrain, exigent que de grandes précautions soient prises au plan méthodologique et que, notamment, les types d'entreprises concernées soient clairement identifiées.

Un premier rapprochement s'impose avec l'enquête réalisée dans 4 villes de l'intérieur ivoirien auprès de 476 petites et micro-activités (la plupart 
informelles) par Benoît Lootvoet (Lootvoet, 1988). Ses résultats sont très proches de ceux de Toumodi. Ainsi, son analyse (Ibidem : 197-198) établit la mesure des différentes combinaisons suivantes dans le domaine de la formation initiale du capital : l'épargne seule atteint $36 \%$ des modalités, le don seul, $29 \%$; le don et l'épargne, $12 \%$; le prêt et l'épargne, $9 \%$; le prêt seul, $8 \%$; le don et le prêt, $5 \%$; cet ensemble couvre donc $99 \%$ des cas. La rareté du crédit bancaire est ici confirmée (Ibidem : 204) : sur les 476 petits établissements 3 seulement ont bénéficié d'un financement bancaire contribuant au capital de démarrage. Le chercheur (p. 205-208) signale que dans 110 des 146 points d'activité (sur 476) caractérisés par des investissements complémentaires, ce sont les bénéfices tirés de l'activité qui en ont permis le financement, les autres modalités, diverses, étant marginales (prêts ou dons familiaux ou amicaux). Les crédits bancaires, quant à eux, n'interviennent partiellement que dans 4 cas.

Une enquête réalisée au Ghana voisin par Paul Kennedy (Kennedy, 1980 : 48 et $\mathrm{s}$.) auprès de 186 entreprises de différentes tailles nous informe que l'épargne personnelle est à l'origine financière exclusive de $55 \%$ de l'ensemble des firmes, les aides et prêts familiaux à l'origine de $33 \%$, les autres sources de financement (prêts d'anciens employeurs, avances des clients, etc.) représentant $12 \%$. L'analyste notait également que les investissements nouveaux réalisés sous forme d'équipement - les seuls qu'il analysait - provenaient pour $55 \%$ des cas de réinvestissements directs de l'exploitation, pour $24 \%$ de prêts commerciaux (plus fréquents pour les grandes entreprises - qui constituaient une fraction de son échantillon - et plus rares pour les petites), enfin pour $21 \%$ d'une épargne personnelle ou familiale constituée en dehors de l'entreprise concernée (plantations, revenus immobiliers, etc.). Les grandes tendances en matière de types de financement des activités sont donc confirmées.

Pour revenir à la Côte-d'Ivoire, nous disposons de deux autres références documentaires précises sur les problèmes de financement des entreprises. La première est l'enquête réalisée sous l'égide du ministère ivoirien du Plan (Baris et Zaslavsky, 1981). L'échantillon était constitué de 41 petites entreprises des secteurs de l'industrie et du BTP. Elles se décomposaient, selon les auteurs, en 18 PME modernes respectant la comptabilité légale; en 16 petites entreprises de transition utilisant une comptabilité rudimentaire, mais employant au moins un salarié permanent et, enfin, en 7 établissements du secteur informel n'ayant recours ni à un début de comptabilité, ni à des salariés. L'échantillon intégrait donc des catégories d'entreprises du type de celles enquêtées à Toumodi. Sur ces 41 petites et moyennes entreprises, 19 étaient localisées à Abidjan. Les principaux enseignements financiers de cette très sérieuse étude se présentent ainsi : en ce qui concerne la formation du capital initial, les auteurs relèvent l'importance générale de l'épargne personnelle, mais notent la tendance des fonds propres à décroître avec la taille de l'entreprise, puisque représentant, en 
part relative de l'investissement initial, $71 \%$ des entreprises informelles, $62 \%$ des entreprises de transition et $31 \%$ des PME modernes. La part des prêts familiaux décroît également avec la taille, correspondant à $25 \%$ des fonds de démarrage dans l'informel, $21 \%$ dans le secteur de transition, $5 \%$ dans les PME modernes. Enfin, la part du crédit extérieur (crédit bancaire et créditfournisseur) varie en sens inverse, ayant tendance à croître avec la taille de l'entreprise (4\% dans l'informel, $17 \%$ dans la transition, $64 \%$ dans les PME). Les auteurs ont relevé un crédit bancaire dans 6 entreprises seulement, toutes des PME (soit $30 \%$ d'entre elles). En matière d'investissements ultérieurs, la part de l'autofinancement s'élevait à $53 \%$ dans les PME, $51 \%$ dans les entreprises du secteur de transition et $6 \%$ dans les entreprises informelles. Le constat de ces réinjections financières dans l'entreprise a conduit légitimement les enquêteurs à militer pour une relativisation de «l'image de l'entrepreneur national qui gaspille l'essentiel de ses bénéfices dans des dépenses ostentatoires en faisant dépérir son entreprise, faute d'investissements de type productif » (Ibidem : 16). Sur ce chapitre des investissements additionnels, les prêts familiaux ou amicaux sont évalués à $90 \%$ dans l'informel, $25 \%$ dans le secteur de transition et $1 \%$ dans les PME modernes. Quant au crédit bancaire, une fois mis à part les financements d'automobiles - dont il est difficile de déterminer la fonction «productive" - ils étaient de $25 \%$ dans les PME, de $5 \%$ dans le secteur de transition et nuls dans l'informel. Encore faut-il préciser que les PME ayant obtenu du crédit institutionnel sont toutes localisées à Abidjan.

C'est précisément cette question spatiale qui se trouve être une des clefs pouvant expliquer des différences sensibles de résultats entre différentes enquêtes, même si elles portent sur des catégories à peu près semblables d'entreprises. Nous en avons un exemple avec l'étude d'une mission d'évaluation réalisée sur 50 PME ivoiriennes en 1986 (République française, 1986). Des disparités assez considérables sont visibles entre les résultats de cette enquête et ceux des études présentées jusqu'à présent. Or, les caractéristiques de structure de la population d'entreprises ayant servi d'échantillon pour cette étude réalisée en 1986 ne sont apparemment guère différentes de celles des petites et moyennes entreprises enquêtées par ailleurs. Les enseignements de l'étude sont pourtant très particuliers. Pour ne retenir que quelques données rapides : 29 des 50 PME enquêtées avaient bénéficié de concours bancaires au moment de leur création. En outre, 7 d'entre elles avaient obtenu le soutien de structures publiques de promotion de l'entrepreneuriat national (OPEI et CAPEN), 16 d'entre elles avaient bénéficié en début d'activité de marchés de l'État et 13 d'entre elles, enfin, avaient établi un partenariat avec des entreprises étrangères. Toutes proportions considérables de nature à particulariser très nettement cet échantillon. Des biais, qui interdisent de généraliser les résultats de cette enquête à l'ensemble de la Côte-d'Ivoire, sont intervenus dans la confection de la liste des entreprises à enquêter. L'échantillon a en effet été constitué à partir de bases 
institutionnelles (Centrale de bilans de la banque des données financières du ministère de l'Économie, ministère du Plan et de l'Industrie, Chambre de Commerce, etc.) et sur la seule agglomération abidjanaise, ce qui a eu pour effet mécanique de faire surreprésenter dans l'échantillon d'enquête les entreprises aidées et soutenues par les diverses structures officielles aux plans administratif, financier, technique parce que proches - politiquement, socialement et géographiquement - des sommets de l'État, des appareils centraux et décisionnels des diverses institutions en cause. D'où, en particulier, un taux de crédit bancaire très élevé et peu représentatif de la situation financière des PME à l'échelle du pays, comme nous l'avons vu précédemment.

Un autre point de repère peut être pris, prudemment encore, avec les résultats observés dans le milieu exclusif des activités informelles. La synthèse réalisée par J.-P. Lachaud (Penouil et Lachaud, $1985: 123$ et s.) porte sur de nombreuses enquêtes faites dans plusieurs pays africains dont la Côte-d'Ivoire, et relatives à des centaines de micro-activités. S'agissant du capital de départ, deux modes principaux de constitution se dégagent : l'épargne personnelle est citée par $\mathbf{7 5 \%}$ des promoteurs et provient, dans $50 \%$ des cas d'activités indépendantes antérieures; l'aide de la famille (prêts et dons) est citée par $25 \%$ d'entre eux. L'auteur note l'absence totale du système bancaire à ce stade de l'investissement initial. Quant aux investissements complémentaires, ils sont considérés comme financés, à $90 \%$, par l'activité elle-même, les $10 \%$ restants provenant des cercles familiaux, la contribution du circuit bancaire étant de nouveau nulle.

Enfin, on relèvera que sur un plan plus général encore, dans leur tour d'horizon intégrant une littérature couvrant l'ensemble de l'Afrique subsaharienne, Page et Steel (1986: 29 et s.) confirment que l'épargne personnelle alimente la majorité des financements des PME africaines, complétés, plus ou moins loin, par les dons et prêts de parents. Ils indiquent que la grande faiblesse des ressources financières accordées par les banques commerciales et les autres organismes financiers publics (qui «atteignent rarement $10 \%$ du capital initial») «constitue une caractéristique frappante » de l'univers des PME africaines.

Les résultats obtenus sur Toumodi ne sont donc pas atypiques. Portant sur des strates particulières d'entreprises (petites entreprises déjà relativement structurées et frange basse des moyennes entreprises), ils rendent compte de la situation financière générale à l'échelle du pays, mais aussi au-delà sans doute, de cette fraction de l'univers entrepreneurial. En résumé, et relativement à la question des parts respectives des financements institutionnels et non institutionnels, on admettra que l'autofinancement constitue le mode très largement dominant de capitalisation des entreprises, que les méthodes informelles qui mettent 
principalement en jeu les contributions lignagères n'ont qu'un rôle accessoire, en tout état de cause inférieur à ce que pourrait laisser penser la quasi-absence du système bancaire officiel dans les fonds de démarrage et dans les investissements ultérieurs des entreprises. L'autofinancement massivement constaté, et synonyme de réinvestissement, témoigne de la force d'un processus d'accumulation qui fait distinguer les établissements enquêtés des installations purement informelles assurant à peine la survie de leurs exploitants.

Quant à la faible contribution des tontines au financement de l'activité économique, elle ne doit pas beaucoup surprendre. La littérature sur les pratiques tontinières a mis l'accent sur la typologie des associations financières, sur leurs modalités de fonctionnement, sur la mathématique des intérêts, etc., mais demeure fort laconique sur la nature des emplois faits des fonds collectés par les participants. On ne trouve nulle mesure des utilisations générales et, a fortiori, dans le cadre du financement entrepreneurial des sommes mobilisées à travers les tontines dans la plupart des travaux spécialisés tels Baulier et alii, 1988 ; Bekolo-Ebe et alii, 1991 ; Dupuy, 1990 ; Issoufou, 1990 ; Lelart et Gnasounou, 1990 ; Nzisabira, 1991 ; Rietsch, 1989 et 1990 ; Tinguiri, 1990, etc. Parmi ceux qui mentionnent - sur déclaration des enquêtés et sans qu'une mesure rigoureuse, d'ailleurs délicate à faire, ne permette d'en contrôler la véracité - des concours tontiniers au développement des affaires, signalons Gnansounou (1991 : 35) qui ne fournit aucun détail et laisse intacte la question d'une contribution à l'entreprise ou à l'entrepreneur, ce qui n'est pas tout à fait la même chose ; Brenner, Fouda et Toulouse (1990a), à propos du cas bien connu des Bamiléké camerounais ; Henry, Tchente et Guillerme (1990) qui évoquent simplement la forte probabilité que les fonds rassemblés « servent également à des financements de trésorerie, soit dans le secteur des PME, soit dans le cadre d'occasions commerciales momentanées [...], il faut signaler cependant qu'aucune enquête n'a pu être réalisée à ce jour sur cette question [...] » (p. 30). Fromain (1990), exploitant 677 réponses d'adhérents et ayant classé les utilisations de fonds en 6 grandes classes, dénombre 11,5\% des réponses se rapportant au «financement d'activités commerciales, artisanales et agricoles » (p. 166), alors que Soedjede (1990, in Lelart) ne peut que faire état d' « intentions » d'utilisation dans le domaine du capital commercial (p. 219).

Au total, il faut bien admettre que la moisson est bien peu substantielle en matière d'évaluation précise du concours tontinier au financement des entreprises : bon nombre d'études ne s'en préoccupent pas; celles qui mentionnent cette fonction ne peuvent que s'en remettre à sa plausibilité, et celles qui tentent de mesurer son étendue prennent les motivations et les intentions pour des actes avérés ou bien ne peuvent aller au-delà de l'enregistrement et de l'exploitation de simples déclarations, sans autre forme de contrôle, lequel suppose, il est vrai, 
proximité, familiarité et durée d'observation. Les difficultés objectives d'évaluation rigoureuse sont souvent rédhibitoires et un expert comme Michel Lelart les a clairement exposées (Lelart, $1991: 13$ et s.). C'est pourquoi on peut partager sa grande prudence lorsqu'il conclut d'un rapide tour d'horizon de la documentation sur la question que "l'épargne des tontines ne sert pas en priorité l'investissement » (p. 15). On pourrait ajouter que l'engouement récent, tout à fait légitime et scientifiquement pertinent, pour les études sur la finance informelle en général et sur les tontines en particulier, faute de déboucher encore sur des mesures précises des emplois des fonds collectés, est propre à donner prise à une surestimation impressionniste du rôle que les circuits parallèles sont à même de jouer dans le soutien et la croissance des petites et moyennes entreprises. Plus généralement encore, ainsi que le notait Philippe Hugon (Hugon, 1990 : 319), les systèmes financiers informels « sont rarement à même de financer des investissements productifs de moyenne et même de faible dimension ».

La situation camerounaise où le phénomène tontinier a servi le dynamisme économique bamiléké, le contexte béninois bourgeonnant de tontines ne peut être généralisé sans grave erreur. En Côte-d'Ivoire aussi, les tontines existent et prospèrent. Mais elles sont pour l'essentiel l'affaire des femmes et remplissent d'autres fonctions (couverture sociale, mutualisme, financement de grosses dépenses ponctuelles de consommation, etc.) que le financement des investissements réalisés au sein des entreprises.

\section{Esquisse compréhensive : des modes de financement à la logique d'accumulation des PME}

Par-delà l'existence de réelles variations généralement corrélées à la taille des entreprises concernées, comment expliquer que la structure des financements leurs types et leur place relative - paraisse s'appliquer dans ses grandes lignes à l'ensemble des établissements ? Comment rendre compte de la faiblesse générale des financements externes des entreprises, qu'ils soient institutionnels ou informels ? Comment même éclairer une situation globalement caractérisée par une sous-utilisation du potentiel des circuits financiers parallèles ? Ces quelques questions et d'autres suggèrent que soit construit un cadre conceptuel permettant d'apporter des éléments de réponse.

Contentons-nous de rappeler quelques directions dans lesquelles des ensembles de notations rendent raison, de fait, des situations observées. Sur l'incompréhension entre entrepreneurs et banquiers, beaucoup a été dit et écrit, et de fort justes choses. Dans une riche littérature, retenons les observations de 
Labazée (1988: 156-162). Il fait état de la circonspection des exploitants du grand commerce traditionnel à l'égard de l'institution bancaire à qui, pourtant, ils confient leurs fonds. Il avance que les taux d'intérêt pratiqués par les établissements financiers ne sont pas le seul fondement de cette méfiance : des décalages de temporalité (court et long terme), la nécessité pour les commerçants d'intervenir rapidement sur les marchés, la complexité, la durée et donc le coût des négociations avec la banque, l'exigence de pièces comptables, les contrôles que s'autorise l'établissement bailleur, les exigences particulières de garanties des prêts accordés, etc. mettent en évidence l'inadéquation aux normes bancaires du fonctionnement du grand commerce, lequel finance, à la fin, ses opérations courantes avec ses ressources propres, et les modifications d'organisation, de gestion et de direction des établissements tenus par les grands commerçants qu'induiraient cette adaptation aux exigences et aux attentes des organismes prêteurs.

De leur côté, Baris et Zaslavsky (1981, tome II : 33-42) ont longuement développé les raisons de l'absence de soutien des banques commerciales au développement des petites et moyennes entreprises : coûts de gestion élevés de prêts réduits mais nombreux, absence de garanties comptables dans l'entreprise, faiblesse des sûretés présentées, incertitude des remboursements, impréparation des entreprises aux conditions et délais de transaction, tendance des établissements financiers à accorder des crédits à court terme en minimisant les risques dans le temps, absence d'autonomie de décision des agences locales et régionales, etc. Ces faits, vérifiés en maintes occasions, expliqueraient la faible participation des banques aux activités de l'artisanat et des PME résumée en 1980 dans cette situation ivoirienne : à l'échelle nationale, les concours bancaires représentaient à peine $1 \%$ du total des crédits dans les PME et les entreprises artisanales du secteur moderne couvertes par la Centrale de bilans.

Symétriquement, les atouts de la finance informelle ont été largement identifiés éclairant certains de ses succès, au demeurant, comme on l'a vu, circonscrits : l'insertion dans un environnement de proximité où le contrôle social réduit les risques de non-remboursement, la faiblesse de ses coûts de gestion, sa flexibilité, son adaptabilité à la diversité des situations, etc. (voir par exemple Hugon, $1990: 319$ et s.).

Une grande partie de la documentation disponible sur la question du financement des PME africaines, de même que bon nombre d'études réalisées sur les circuits financiers informels font, explicitement pour certaines, implicitement pour d'autres, dépendre le développement et la prospérité des petites et moyennes entreprises du facteur financier d'une offre suffisante et adaptée de moyens financiers aux besoins des entreprises, d'un plein épanouissement des potentialités des circuits parallèles. En tout état de cause, l'accroissement des 
instruments de crédit serait nécessaire et viendrait judicieusement soutenir l'expansion des activités entrepreneuriales. Il est possible que cette perspective ne soit pas tout à fait exacte et que les présupposés sur lesquels elle est fondée ne résistent pas à l'analyse.

Pour développer ce point de vue, on peut s'appuyer tout d'abord sur les corrosives et pertinentes observations d'Ernest Aryeetey faites à la suite d'enquêtes auprès d'environ 300 petites entreprises du Ghana (Aryeetey, 1991). Le chercheur épingle quelques postulats sous-jacents à de nombreux travaux et quelques malheureuses simplifications : le financement informel serait le substitut automatique et idéal au crédit institutionnel rationné ou absent ; le développement des financements informels serait la condition d'un plus grand dynamisme des PME; à terme, en contribuant à la création d'un environnement plus favorable, l'augmentation du crédit en général, et du crédit informel en particulier, permettrait un accroissement de la taille des entreprises et le passage d'établissements artisanaux ou semi-industriels à un stade industriel, etc. Les analyses qui se font l'écho des «demandes » et autres «besoins » de crédit enregistrés auprès des petits entrepreneurs ne sont pas sans ambiguité ; bon nombre de leurs problèmes financiers ne sont pas nécessairement liés à des demandes de crédit même si cette liaison est souvent évoquée : il s'agit souvent, en fait, de créances non recouvrées, quelquefois de produits inadaptés à la demande et, plus largement, d'une mauvaise adéquation aux conditions du marché souvent vécus, il est vrai, en termes financiers; en outre, l'aide appelée par les petits entrepreneurs est plus limitée qu'on ne le croit trop souvent : c'est la trésorerie plus que le long terme qui est en cause. E. Aryeetey a constaté, au cours de ses enquêtes de terrain, que toutes les possibilités de crédit informel étaient loin d'être utilisées par les petits entrepreneurs. Enfin, lorsque ceux-ci y ont recours, c'est bien plutôt pour financer leur consommation privée, ou même pour réaliser ponctuellement une opération spéculative que pour consolider financièrement l'activité de leur entreprise. Le chercheur conclut que ces liaisons sont, pour l'heure, plus postulées que démontrées, que les réalités du terrain entrepreneurial au Ghana sont loin de vérifier la validité de toutes les assertions prononcées en matière de financement informel des petites entreprises et qu'il faut être, en la matière, d'une grande prudence. La consolidation du tissu des entreprises, leur prospérité et leur développement ne se réduisent pas par des termes aussi simplificateurs que «besoins de crédit informel ».

Les débats portant sur l'ampleur et la nature de l'accumulation dans le secteur des petites entreprises et des micro-activités informelles peuvent aider à avancer encore davantage dans la mise en place du cadre conceptuel propre à répondre aux questions initialement posées. Les réflexions faites par B. Lootvoet dans le cadre de vigoureuses remarques méthodologiques sur la mesure de l'accumulation et de l'examen des enjeux de politique économique sont des plus 
profitables (faut-il, ou encore, peut-on aider par des mesures appropriées le dynamisme des petites entreprises les plus performantes ?) (Lootvoet, 1988, p. 209-218). Passant en revue diverses thèses et divers courants de pensée sur la question de l'accumulation et le type de reproduction - simple ou élargie - qui prévaut dans le monde des petites activités urbaines et résumant les résultats de ses propres enquêtes, Lootvoet y vérifie à la fois l'existence d'une accumulation de capital saisissable non plus par une approche de micro-économie mais, nouveauté, de macro-économie et précise la nature de cette accumulation : elle ne se traduit pas nécessairement par la croissance du capital productif au sein de chaque établissement en question; des investissements tout autant productifs sont réalisés au-dehors et on constate notamment une tendance à la multiplication des points d'activité. C'est ce qui explique, entre autres, le décalage entre la taille du capital accumulé par le petit entrepreneur et la modicité des investissements complémentaires qu'il réalise au sein de l'entreprise. Ainsi peut-on mieux comprendre quelques constats : l'élévation des revenus tirés d'une activité ne débouche nullement sur une tendance à l'accumulation de type intensif reposant sur un accroissement du capital productif de l'établissement en question ; la taille des établissements artisanaux et commerciaux tend à se stabiliser dans le temps.

L'analyse de la dynamique de l'investissement que propose $\mathrm{X}$. Oudin à partir de ses résultats d'enquête sur la branche textile du secteur informel à Niamey conforte cette problématique et apporte une compréhension accrue du processus d'accumulation (Oudin, 1990). Ce chercheur a éclairé deux paradoxes qui rendent perplexes les experts et peuvent expliquer les défaillances des politiques d'assistance aux entrepreneurs nationaux : d'une part, l'apport de capital financier n'a qu'une incidence indirecte sur le niveau de production; d'autre part, des entreprises qui paraissent stagner génèrent des revenus suffisants pour financer par ailleurs toutes sortes d'investissements hors de ces entreprises elles-mêmes. Les données d'enquête montrent que le mouvement d'investissement se réduit la plupart du temps à la seule phase de démarrage de l'activité et que, pour le reste, les pratiques d'investissement se limitent à l'entretien de l'appareil de production sans avoir pour vocation l'augmentation du capital fixe. Il n'existe pas, d'autre part, de relations entre les résultats des entrepreneurs enquêtés et l'importance de l'investissement. X. Oudin confirme la hiérarchie déjà évoquée des sources de financement (épargne personnelle, prêts familiaux et communautaires, inexistence des prêts bancaires - alors même que $11 \%$ des enquêtés ont des comptes bancaires - et rareté du recours au système tontinier). Analysant les dépenses et les placements réalisés par les petits entrepreneurs, s'appuyant sur l'écart entre la croissance du secteur textile informel et la faiblesse des résultats apparents, l'auteur avance une interprétation qui rend compte de la dynamique particulière du secteur (importance des 
nouveaux entrants, disparitions massives, croissance globale du nombre d'établissements) et la nature de l'accumulation qui y est opérée. Les entrepreneurs dégagent une épargne peu visible qu'ils convertissent en de multiples formes sans rapport direct avec l'activité en question (prêts, dons, placements spéculatifs, stockage de nourriture, etc.). Ils opèrent également une diversification de leurs activités par la création de nouveaux établissements et des transferts intersectoriels (taxis, maraîchage, immobilier, commerce, etc.). Cette accumulation est saisissable, comme le montrait précédemment Lootvoet, à l'échelle macro-économique des secteurs concernés. Il s'ensuit que l'erreur des programmes officiels d'assistance est de reposer implicitement sur une hypothèse évolutionniste (l'artisan serait le prototype du chef d'une plus grande entreprise) et de privilégier le crédit d'investissement en facilitant l'acquisition de capital. Ceci conduit à une tendance à la surcapitalisation des entreprises aidées et à une baisse de productivité du capital. Or, nous dit Oudin, le financement des investissements n'est pas le principal problème des petits entrepreneurs, mais bien plutôt la taille des marchés auxquels ils ont accès.

La pertinence de ces analyses se présente, nous semble-t-il, sous un double aspect : en premier lieu, elles rendent compte de phénomènes empiriquement observables; d'autre part, en précisant la nature réelle de l'accumulation, elles permettent de mieux situer les divers problèmes des entreprises africaines et de relativiser les facteurs financiers.

De nombreuses observations de terrain confirment ou s'inscrivent dans le droit prolongement des propos précédents. Le monde des entreprises est fortement segmenté et prend en défaut toute analyse et toute politique économique fondées sur un présupposé continuiste. Les micro-activités tendent à demeurer dans le même secteur informel, les petites entreprises ont vocation à rester petites et les moyennes à rester moyennes. Rares sont les exemples de passage de taille réalisé et, a fortiori, réussi. De très nombreuses études ont montré que les PME ne sont pas d'anciens établissements informels et que l'ingénieux et dynamique artisan n'est pas la préfiguration historique de l'exploitant d'une beaucoup plus grande entreprise. Cette segmentation s'explique elle-même à la fois par l'existence de seuils (financiers, techniques, organisationnels, culturels, etc.) difficiles à franchir et par les diverses logiques qui président à la gestion des entreprises et qui orientent les types d'accumulation qui y prévalent ; c'est pourquoi, entre autres, nous proposons de bannir le terme d'«activités de transition » pour désigner les établissements informels intermédiaires entre ceux qui stagnent et ceux qui dégagent des surplus à cause de ces connotations évolutionnistes contredites par l'expérience. L'accumulation extensive, engendrée par la diversification des activités, que l'on observe à la fois dans le secteur informel et dans les classes petites et moyennes des 
entreprises plus structurées, voire modernes, s'appuie sur des déterminants précis (contrôle de la main-d'œuvre, difficultés à maîtriser un marché élargi, absence de soutien des structures officielles, etc.), et l'ensemble de ces facteurs clairement intériorisés par les petits entrepreneurs font de l'expansivité un processus fondé en rationalité.

Ce ne sont donc pas forcément les problèmes de capitaux qui limitent l'expansion des firmes, et ce n'est donc pas nécessairement le développement des instruments financiers institutionnels ou non institutionnels qui va dynamiser les entreprises et rendre plus fluides et plus fréquents les passages de taille. Audelà du monde des tout petits métiers, d'évidents surplus sont dégagés, des réinvestissements sont effectués, mais dans des limites et sous des contraintes de structure qui n'appellent pas l'explosion des services bancaires et des systèmes financiers informels. On comprend mieux, dans une telle perspective, que les besoins financiers se trouvent relativisés, essentiellement satisfaits par la mobilisation des ressources propres et complétées, çà et là, par des concours extérieurs, rares en ce qui concerne les circuits institutionnels et de faibles volumes lorsqu'il s'agit des réseaux informels. On voit aussi en quoi le développement entrepreneurial semble moins conditionné par le capital que par l'accès et l'adaptation à des marchés de plus grande taille. On ne gagnerait rien à laisser perdurer cette double illusion qui voudrait que la finance en général, la finance informelle en particulier, soient les seuls ou même les principaux facteurs limitants dans la vie des PME africaines.

\section{Bibliographie}

ARYEETEV, E. (1991), « How important are formal and informal external finance to microentreprise development in Ghana, Communication en vue du séminaire international », Finance et développement rural en Afrique de l'Ouest, 21-25 octobre, multig., 25 p.

Barbier, J.-P., C. Courlet et R. TyberghiEn (1986), « Émergence et développement des petites entreprises en Afrique au Sud du Sahara ; résultats d'une enquête effectuée au Cameroun ", Caisse centrale de coopération économique, Notes et études, $n^{\circ} 6$.

Baris, P. et J. ZaSLAvSKY, (1981), « Les stratégies d'investissement des petits industriels nationaux et leurs problèmes de financement », Ministère du Plan et de l'Industrie, multig., 2 tomes, Abidjan.

BAULIER, F. et alii (1988), « Les tontines en Afrique, rôles et évolutions », Caisse centrale de coopération économique, Notes et études, $\mathrm{n}^{\circ} 12$. 
Beкolo-Ebe, B. et alii (1991), « Enquête sur les tontines dans les provinces du Centre, du Littoral, de l'Ouest et du Nord-Ouest », UREF/AUPELF, Notes de recherche du Réseau entrepreneuriat, $\mathrm{n}^{\circ}$ 91-22.

Brenner, G., H. Fouda et J.-M. Toulouse (1990a), « Les entrepreneurs Bamiléké de Douala et leur entreprise ", UREF/AUPELF, Notes de recherche du Réseau entrepreneuriat, $\mathrm{n}^{\circ}$ 90-6.

Brenner, G., H. Fouda et J.-M. Toulouse (1990b), « Les tontines et la création d'entreprises au Cameroun », dans G. Hénault, et R. M'Rabet (1990), p. 97-105.

Charmes, J. (1990), « Une revue critique des concepts, définitions et recherches sur le secteur informel » dans D. Turnham, B. Salomé, et A. Schwartz (éd.), Nouvelles approches du secteur informel/The Informal Sector Revisited, OCDE/OECD, Paris, p. 11-51.

Dromain, M. (1990), « L'épargne ignorée et négligée. Les résultats d'une enquête sur les tontines au Sénégal », dans M. Lelart (éd.), La tontine pratique informelle d'épargne et de crédit dans les pays en voie de développement, AUPELF et John Libbey Eurotext, p. 139-176.

DupuY, C. (1990), «Les comportements d'épargne dans la société africaine : études sénégalaises », dans M. Lelart (éd.), op. cit., p. 31-51.

FAURE, Y.-A. (1989), « Le monde des entreprises en Côte-d'Ivoire, sources statistiques et données de structure », UREF/AUPELF, Notes de recherche du Réseau entrepreneuriat, $n^{\circ} 89-1$.

FAURE, Y.-A. (1991), « Entrepreneurs d'Afrique subsaharienne : communautés entrepreneuriales dans les travaux anglophones (Ghana, Nigéria, Kenya, etc.) et comparaisons avec la Côte-d'Ivoire », UREF/AUPELF, Notes de recherche du Réseau entrepreneuriat, $\mathrm{n}^{\circ}$ 91-19.

FAURÉ, Y.-A. (1992), « Financement de la petite et moyenne entreprise à Toumodi (Côted'Ivoire) : l'illusion informelle », UREF/AUPELF, Notes de recherche du Réseau entrepreneuriat, $n^{\circ}$ 92-25.

Gnansounou, S. (1991), « L'épargne informelle et le financement de l'entreprise productive, référence spéciale aux tontines et à l'artisanat béninois ", UREF/AURELF, Notes de recherche du Réseau entrepreneuriat, $n^{\circ}$ 91-20.

HAUDEVILle, B. (1990), «Épargne informelle et financement de l'entreprise productive », dans G. Hénault et R. M'Rabet (1990), p. 77-85.

Henault G. et R. M'Rabet (éd.) (1990), « L'entrepreneuriat en Afrique francophone : culture, financement et développement ", Actes des journées scientifiques du réseau «Financement de l'entrepreneuriat et mobilisation de l'épargne » tenues les 16 et 17 février 1989 à Casablanca, AUPELF et Paris, John Libbey Eurotext. 
Henry, A., G.H. Tchente et P. Gullerme, (1990), « La Société des Amis, étude des tontines à enchères du Cameroun ", Caisse centrale de coopération économique, Notes et études, $\mathrm{n}^{\circ} 34$.

Hugon, P. (1990), « La finance non institutionnelle : expression de la crise du développement ou de nouvelles formes de développement ? ", dans M. Lelart (éd.), op. cit., p. $309-321$.

Hugon, P. (1988), « Les politiques d'ajustement et les micro-entreprises dans les pays en développement », Caisse centrale de coopération économique, Notes et études, $n^{\circ} 13$.

Issoufou, S. (1990), « Le phénomène tontinier au Burkina Faso, étude sur 69 cas », UREF/AUPELF, Notes de recherche du Réseau entrepreneuriat, $n^{\circ}$ 90-12.

KenNEDy, P. (1980), Ghanaian Businessmen. From Artisan to Capitalist Entrepreneur in a Dependent Economy, München/London, Weltforum Verlag.

LabazeE, P. (1988), Entreprises et entrepreneurs du Burkina Faso, Paris, Karthala.

LACHAUD, J.-P. (1985), « Les activités informelles urbaines en Afrique subsaharienne », dans M. Penouil et J.-P. Lachaud, Le développement spontané, Paris, Pédone, p. 104-147.

LELART, M. (éd.) (1990), La tontine, pratique informelle d'épargne et de crédit dans les pays en voie de développement, AUPELF et Paris, John Libbey Eurotext.

LelarT, M. (1990), «Une tontine mutuelle dans l'administration béninoise », dans M. Lelart, (éd.), op. cit., 1990, p. 53-80.

LeLART, M. (1991), « Les tontines et le financement de l'entreprise informelle », UREF/ AUPELF, Notes de recherche du Réseau entrepreneuriat, $n^{\circ}$ 91-18.

Lelart, M. et S. Gnansounou (1990), « Tontines et tontiniers sur les marchés africains : le marché Saint-Michel de Cotonou », dans M. Lelart (éd.), op. cit., p. 109-138.

Le PAPE, M. (1983), « De l'indigène à l'informel », Cahiers d'Études africaines, $\mathrm{n}^{\circ} 89-90$.

LOOTVOET, B. (1988), "L'artisanat et le petit commerce dans l'économie ivoirienne », Paris, Éditions de l'ORSTOM, série « Études et thèses » (première publication : «Contribution de l'artisanat et du petit commerce à l'économie ivoirienne», thèse de $3^{\mathrm{e}}$ cycle en économie, Université de Clermont I et CERDI, 1986).

Miras, C. de (1987), « De l'accumulation de capital dans le secteur informel », Cahiers des Sciences humaines, Paris, ORSTOM, ${ }^{\circ} 1$, p. 49-74.

NzISABIRA, J. (1991), "Les associations tontinières, mouvement associatif, épargne et entrepreneuriat au Rwanda », UREF/AUPELF, Notes de recherche du Réseau entrepreneuriat, $\mathrm{n}^{\circ}$ 91-15. 
Oudin, X. (1990), «Dynamique de l'investissement dans le secteur informel : une étude de cas au Niger », dans G. Hénault et R. M'Rabet, op. cit., p. 145-155.

Oudin, X. (1985), «Les activités non structurées et l'emploi en Côte-d'Ivoire », Thèse de sciences économiques, Université de Rennes.

PAGe, J.M. et W.F. Steel (1986), « Le développement des petites entreprises, questions économiques tirées du contexte africain ", Washington, Banque mondiale, Document technique $n^{\circ} 26 \mathrm{~F}$.

Pairault, T. (1990), « Sociétés de tontines et banques des petites et moyennes entreprises à Taiwan », dans M. Lelart (éd.), op. cit., p. 281-308.

RÉPuBlique fRANÇAISE, Ministère des Relations Extérieures (1986), " Analyse ex-post de la promotion des PME et de l'artisan en Côte-d'Ivoire », Paris, Mission d'évaluation SEDES/CECOF, multig., pag. multi.

RIETSCH, C. (1990), « Les paramètres de base des tontines à Niamey », UREF/AUPELF, Notes de recherche du Réseau entrepreneuriat, $n^{\circ}$ 90-8.

RIETSCH, C. (1989), « Une tontine à double niveau d'enchères », UREF/AUPELF, Notes de recherche du Réseau entrepreneuriat, $n^{\circ}$ 89-5.

SoEdjedE, D.A. (1990), « L'épargne et le crédit non structurés au Togo », dans M. Lelart (éd.), op. cit., p. 203-237.

SoEDJEDE, D.A. (1990), « Politique de financement de l'entrepreneuriat au Togo », dans G. Hénault et R. M'Rabet, op. cit., p. 129-144.

Tinguiri, K.L. (1990), « Épargne et crédit informels en milieu rural au Niger : l'activité des tontines et des gardes-monnaie villageois ", dans M. Lelart (éd.) op.cit., p. 177-202.

Weber, M. (1964), L'éthique protestante et l'esprit du capitalisme, Paris, Plon, (1 $1^{\text {re }}$ dition française). 